\title{
Mesh Plug Migration After a Robotic Inguinal Hernia Repair
}

\author{
Jasneet Singh Bhullar, ${ }^{a, b}$ Ravi Kapadia ${ }^{a}$, Sumet Silapaswan ${ }^{a}$
}

\begin{abstract}
Even though inguinal hernia repairs are one of the most common surgery but the techniques have been evolving over the years. The evolution has been from open to minimally invasive surgery. Recently robotic surgery has gained popularity and is now well accepted for surgeries of the pelvis and the prostate. Inguinal hernias are detected in $20-30 \%$ of patients undergoing robotic radical prostatectomy, and are concomitantly repaired. A 50-year-old male presented with a non reducible groin mass. He had a prior history of a robotic prostatectomy with a concurrent mesh plug repair of direct inguinal hernia repair three years ago. Preoperative workup revealed mesh plug migration and intra operatively, it was noted that the robotically placed pre-peritoneal mesh plug had migrated through the internal ring into the inguinal canal. Excision of the plug with an open mesh repair of the inguinal hernia was done. A high degree of suspicion helped in correct management of the patient. When dealing with a patient with a prior history of robotic pelvic surgery who presents with a groin mass, mesh migration should be a part of the differential diagnosis. Pre-operative imaging in this select group of patients can aid in making the correct diagnosis and management plan. To the best of our knowledge this is the first report of a mesh plug migration after a robotic inguinal hernia repair.
\end{abstract}

Keywords: Mesh; Mesh plug; Inguinal hernia; Robotic repair; Mesh migration; Robotic inguinal hernia repair

\section{Introduction}

Inguinal hernia repair is one of the most common opera-

Manuscript accepted for publication May 24, 2013

${ }^{a}$ Department of Surgery, Providence Hospital and Medical Centers, Southfield, MI, USA

${ }^{\mathrm{b}}$ Corresponding author: Jasneet Singh Bhullar, Department of Surgery, Providence Hospital and Medical Centers, 16001 West Nine Mile Road, Southfield, MI 48075, USA. Email: drjsbhullar@gmail.com

doi: http://dx.doi.org/10.4021/jcs186w tions, performed by general surgeons in the United States annually. Inguinal hernias can occur unilaterally or bilaterally and can recur after surgery, necessitating reoperation [1]. Amongst the many operative approaches, a single gold standard method for inguinal hernia repair remains a controversial issue. The open technique is a conventional method of repair and includes techniques named after their pioneering surgeons, Shouldice, Bassini and McVay. Each of the operative techniques has shortcomings such as post-operative pain, recurrence and mesh migration. With minimally invasive surgery becoming increasingly popular worldwide, laparoscopic hernia repair has gained widespread acceptance amongst surgeons [2]. Small incisions are made for the operating instruments and laparoscope, and a prosthetic mesh is used to close the hernia defect. There are two approaches for laparoscopic repair, TEP (total extraperitoneal) and TAPP (Transabdominal preperitoneal), with the main variation being whether or not the peritoneal cavity is entered. Laparoscopic repair is associated with a faster recovery to normal activities and less persisting pain and numbness [2]. There also appear to be fewer cases of wound infection and hematoma. However, operating times are longer and there appears to be a higher rate of serious complications in respect of visceral (especially bladder) injuries. Mesh infection is

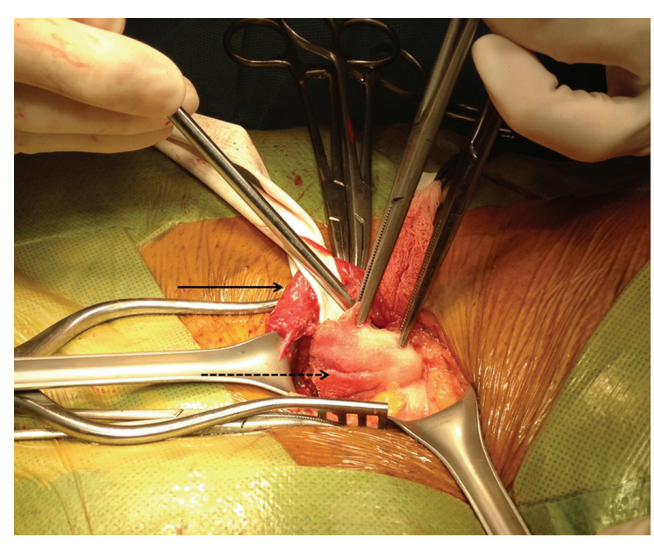

Figure 1. The mesh plug (marked with a black interrupted arrow) in the inguinal canal and attached to the cord structures (marked with a black arrow). 


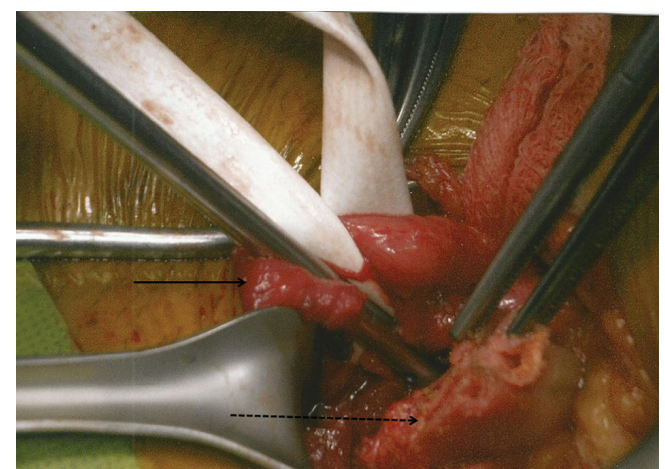

Figure 2. The mesh plug (black interrupted arrow) being dissected out from the dense adhesions between the cord structures (marked with a black arrow) and surrounding tissues.

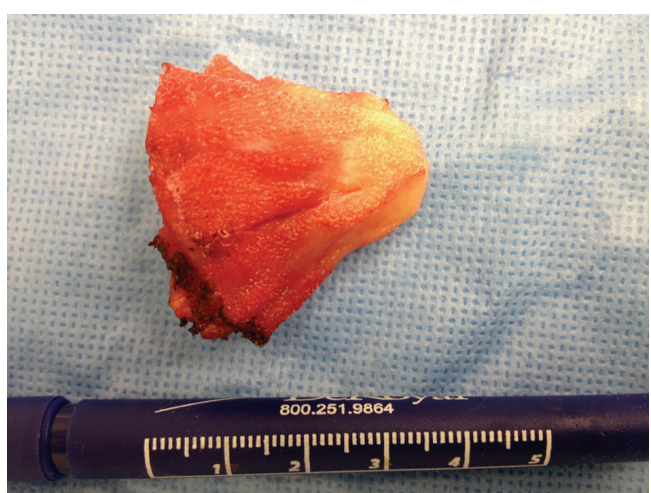

Figure 3. The excised mesh plug.

indirect hernia was repaired using a non absorbable mesh. He was discharged home the day of surgery in stable condition. At a one year follow up the patient continues to do well.

\section{Discussion}

Hernia surgery is an ever evolving surgical procedure. However, in spite of many years of research a gold standard for inguinal hernia repair does not exist. Each technique has its own limitations and complications which are feared by the surgeon. Minimally invasive laparoscopic methods of hernia repair have gained increasing popularity. Although this method decreases hospital length of stay and affords a faster recovery, recurrence is more common than after open repair of primary hernias [3]. A higher reoperation rate following laparoscopic repair is seen in the first couple of years following the initial surgery.

These days hernia repairs are also being performed robotically [5]. This is a relatively new approach, which is gaining popularity due to the widespread use robotic surgery for prostate and pelvic surgery. While robotic surgery offers the patients a minimally invasive operation, it offers the surgeon improved dexterity and visualization [6]. Many inguinal hernia repairs are being performed concomitantly with robotic radical prostatectomy [7]. This approach however does have some limitations and complications with edema and hematoma of the penis being the most common. Also it does not allow the surgeon to control bleeding because of the large diameter of the instruments $(8 \mathrm{~mm})$ and limited number of robotic arms (three) [7]. There has been no standardization of robotic inguinal hernia repair, though use of sutures and mesh plugs have been described. Our patient had undergone a concurrent robotic prostatectomy with an inguinal hernia repair with a mesh plug. Retrospectively, this may not have been the most effective approach, because over time there was migration of the mesh plug from the internal ring into the inguinal canal. In the case of our patient, he did remember that inguinal hernia repair was done with mesh during his 
prostatectomy and his physical examination finding of a hard incarcerated hernia prompted an ultrasound examination.

With more such cases being performed we can expect to see more complications of this method in the near future. Many patients do not have their complete prior operative record as the robotic surgery is done by different specialties and even at other hospitals. It is imperative for the surgeon to consider previous robotic repair for the inguinal hernia too when dealing with a patient with inguinal hernia who had prior robotic surgery of the pelvis. Pre operative imaging in this select group of patients can help in making the correct pre operative diagnosis.

\section{Conclusion}

To the best of our knowledge this is the first report of a mesh plug migration after a robotic inguinal hernia repair. While dealing with a patient with a prior history of robotic pelvic surgery presenting with an inguinal hernia, mesh migration should be a part of the differential diagnosis as patients may not have all their complete operative records. A high degree of suspicion and pre operative imaging can be helpful in planning a successful surgery and avoiding an intra operative surprise.

\section{Disclosures}

All the authors have nothing to disclose.

\section{References}

1. Huang CS, Huang CC, Lien HH. Prolene hernia system compared with mesh plug technique: a prospective study of short- to mid-term outcomes in primary groin hernia repair. Hernia. 2005;9(2):167-171.

2. Neumayer L, Giobbie-Hurder A, Jonasson O, Fitzgibbons R, Jr., Dunlop D, Gibbs J, Reda D, et al. Open mesh versus laparoscopic mesh repair of inguinal hernia. $\mathrm{N}$ Engl J Med. 2004;350(18):1819-1827.

3. Suradom C, Palaphun J. The usage of two umbrella made-mesh plugs in herniorrhaphy: comparative study with Bassini and Lichtenstein method. J Med Assoc Thai. 2011;94(11):1373-1379.

4. Novaretti JP, Silva RD, Cotrim CA, Souto LR. Migration mesh mimicking bladder malignancy after open inguinal hernia repair. Hernia. 2012;16(4):467-470.

5. Finley DS, Rodriguez E, Jr., Ahlering TE. Combined inguinal hernia repair with prosthetic mesh during transperitoneal robot assisted laparoscopic radical prostatectomy: a 4-year experience. J Urol. 2007;178(4 Pt 1):1296-1299; discussion 1299-1300.

6. Stolzenburg JU, Rabenalt R, Dietel A, Do M, Pfeiffer H, Schwalbe S, Dorschner W. Hernia repair during endoscopic (laparoscopic) radical prostatectomy. J Laparoendosc Adv Surg Tech A. 2003;13(1):27-31.

7. Madi R, Daignault S, Wood DP. Extraperitoneal v intraperitoneal robotic prostatectomy: analysis of operative outcomes. J Endourol. 2007;21(12):1553-1557. 